\title{
Verification of chemical evolution of RNA under hydrothermal environments on the primitive Earth
}

\author{
Kunio Kawamura ${ }^{1, a}$, Laura Da Silva ${ }^{2}$, Mari Ogawa ${ }^{3}$, Noriko Konagaya ${ }^{4}$, and Marie-Christine Maurel ${ }^{2}$ \\ ${ }^{1}$ Hiroshima Shudo University, Department of Human Environmental Studies, 1-1-1 Ozuka-higashi, \\ Asaminami-ku, Hiroshima 731-3195, Japan \\ ${ }^{2}$ Institut de Systématique, Evolution, Biodiversité (ISYEB), UMR 7205 CNRS MNHN UPMC EPHE, \\ Muséum National d'Histoire Naturelle, Sorbonne Universités, CP. 50, 57 rue Cuvier, 75005 Paris, \\ France \\ ${ }^{3}$ Yasuda Women's University, Department of Primary Education, 6-13-1 Yasuhigashi, Asaminami-ku, \\ Hiroshima 731-0153, Japan \\ ${ }^{4}$ Yasuda Women's University, Department of Nutritional Sciences, 6-13-1 Yasuhigashi, \\ Asaminami-ku, Hiroshima 731-0153, Japan
}

\begin{abstract}
The RNA World hypothesis proposes that primitive forms of life used polymers resembling RNA both as catalysts and as carriers of genetic information. It has also been suggested that the origin of life occurred in hydrothermal conditions, but this implies that the ester bonds of nucleic acids are sufficiently stable to survive in aqueous conditions at elevated temperatures. Here, we summarize the results of experimental tests of RNA in simulated hydrothermal conditions in which stability is monitored at elevated temperatures and pressures. This perspective provides insight into the evolutionary pathway from small nucleotides to functional RNA molecules and the feasibility of RNA-based life.
\end{abstract}

\section{Simulation experiments of prebiotic RNA formation}

A major unsolved question in origins of life research concerns the process by which nucleic acids were first assembled and incorporated into the earliest forms of life. Substantial evidence supports the conjecture that the first living organisms passed through a phase in which RNA served both as a catalyst and as a carrier of genetic information [1-4]. However, it is still unclear how non-biological processes could have synthesized random polymers of RNA-like molecules as a first step toward living systems. The formation of RNA molecules is required not only for the accumulation of RNA but also for RNA to evolve biochemical functions.

Several studies simulating the prebiotic conditions on the early Earth demonstrated that RNAlike molecules can be synthesized from chemically activated monomers. Because polymerization of nucleoside 5'-triphosphate is not efficient under these conditions, activated nucleoside 5'monophosphorimidazolides were used instead, both in the presence and absence of a polynucleotide template [5-8] (Fig. 1). The formation of oligonucleotides proceeds from the activated nucleotide monomers in the presence of a metal ion catalyst [6] or a clay mineral catalyst [7]. The formation of oligonucleotides in the presence of such prebiotic catalysts leads to the production of oligomers as

a Corresponding author: kawamura@shudo-u.ac.jp

This is an Open Access article distributed under the terms of the Creative Commons Attribution License 4.0, which permits unrestricted use, distribution, and reproduction in any medium, provided the original work is properly cited. 
Metal-catalyzed reaction

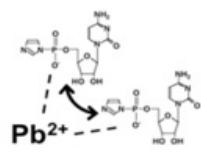

\section{Cyclization}

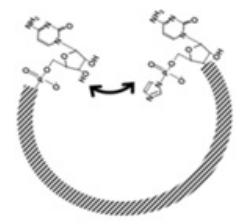

Clay-catalyzed reaction

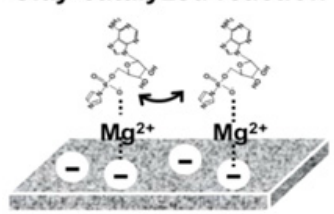

Template-directed reaction

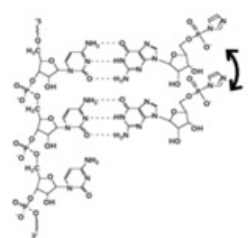

Figure 1. RNA formation models with and without activated nucleotide monomers.

long as 50-mers in the presence of the clay mineral catalyst [8]. It is assumed that the accumulation of oligonucleotides possessing lengths comparable to small biological RNA molecules could have undergone selection and evolution into a variety of biochemical functions $[9,10]$.

The initial experiments demonstrating template-directed formation of oligonucleotides from activated nucleotide monomers were carried out by Orgel and coworkers [11, 12]. These studies showed that guanosine 5'-phosphorimidazolide or guanosine 5'-phospho-2-methylmidazolide forms oligonucleotides up to 40 residues in the presence of a polycytidylic acid template. However, the template-directed reaction does not proceed from activated pyrimidine nucleotide monomers in the presence of a complementary purine polynucleotide template. Thus, the complete replication of all oligonucleotide compositions remains an open question [13, 14].

On the other hand, the conditions on the primitive Earth are unclear. For instance, it is proposed that giant impacts during the late heavy bombardment could have had a sterilizing effect by dramatically increasing global temperatures [15]. In contrast, the primitive Earth may have been completely frozen because of lower solar luminosity [16]. Despite this uncertainty, it is clear that at some point global temperatures were such that a liquid ocean was present with emerging volcanic land masses resembling Hawaii and Iceland today. Recent prebiotic simulation experiments on the formation of biomolecules suggest that hydrothermal environments would have played important roles in the emergence of primitive life-like systems [17]. Several environments have been proposed as plausible sites for the Origins of life, such as a warm pond as already suggested by Charles Darwin in 1871, as well as primeval oceans, rocks and clays, ice, deep sea hydrothermal vents, surface hydrothermal sources etc. Temperature is unquestionably an important factor that must be verified concerning the occurrence of an RNA-based life-like system and the RNA world. However, simulation experiments for the formation of RNA and the template-directed reaction have been applied under mild conditions. Consequently, simulated hydrothermal experiments for the formation and the self-replication of oligonucleotides should be re-examined under a variety of extreme conditions.

\section{Approaches monitoring reactions at elevated temperatures and pressure}

The RNA world hypothesis has been extensively evaluated using our hydrothermal flow reactor systems to solve the compatibility of the hydrothermal origin-of-life hypothesis and the RNA world hypothesis [14, 18]. A real-time monitoring method and an in situ UV-visible absorption 


\section{ORIGINS}

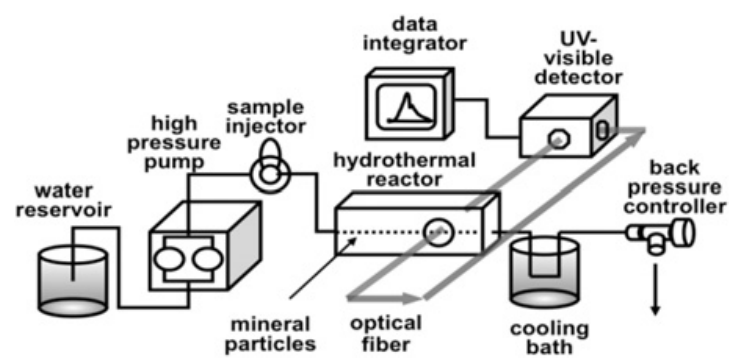

Figure 2. Hydrothermal flow reactor system using narrow tubing. This system enables real-time monitoring and in situ UV-visible monitoring with and without solid particles. Solid particles are packed in the tubular reactor.

spectrophotometric method for hydrothermal reactions within the millisecond to second time-scale at temperatures up to $400{ }^{\circ} \mathrm{C}$ [19] (Fig. 2) provides the information regarding the reaction behaviour, stability, three-dimensional structure, and solubility of nucleotide bases, nucleotides, oligonucleotides, and a hammerhead ribozyme [19-21]. Recently, the technique was applied to monitor hydrothermal reactions of heterogeneous reactions in the presence of naturally-occurring minerals [22]. Highpressure measurements, another powerful tool for the detailed investigation of RNA behavior and biochemical functions of hairpin ribozymes and related chemicals have been successfully performed $[23,24]$. Thermodynamic factors determined through high-pressure measurements clarified the volume changes associated with the conformational modifications on the catalytic pathways. Accumulation of fundamental data of RNA molecules at high temperature and pressure has led to demonstrate the possibility and diversity of RNA-based life-like systems and the RNA world under extreme environments on the primitive Earth.

\section{The accumulation of RNA oligomers under hydrothermal conditions}

One may deny the possibility of the existence of a RNA world under hydrothermal environments on the basis of the qualitative understanding that RNA molecules should normally be weak at high temperatures from the viewpoint of thermodynamics. However, a hydrothermal surface site is a plausible area for the origins of life, providing numerous advantages. Hydrothermal processes concentrate reactants by evaporation, and low water activity with elevated temperatures provide the activation energy necessary for condensation reactions to occur. Small pools produced by hydrothermal conditions associated with volcanic activity are present today and were presumably ubiquitous on the early Earth. Field studies in analogous environments in Iceland, Hawaii, and northern California have shown that the temperature of such pools ranges from $60{ }^{\circ} \mathrm{C}$ to near boiling [25]. They are acidic, between $\mathrm{pH} 2$ and 3. Hydrationdehydration (HD) cycles occur around the edges of the pools in time frames of minutes to hours, and the pools themselves evaporate and refill over weeks to months. Therefore, coupled to the hot springs hydrothermal environment as a plausible site for the origins of life and the "RNA world" hypothesis, we have developed, in collaboration with David Deamer, a simple laboratory simulation of HD cycles which we consider to be an essential factor driving polymerisation of biological monomers. We have tested whether a mixture of ordinary nucleotides when exposed to these simulated prebiotic conditions can be driven toward polymerisation.

It was shown that in these conditions and in the presence of phospholipids, polymers resembling RNAs ranging from 10 to 100 nucleotides were synthesized with a yield of 6\% [26]. We extended this simulation to conditions in which mononucleotides are exposed to HD cycles in the presence of monovalent salts [27]. Ammonium chloride had a high catalytic effect on the polymerisation process allowing synthesis of RNA-like polymers ranging from 10 to 300 nucleotides. During dehydration, salt 


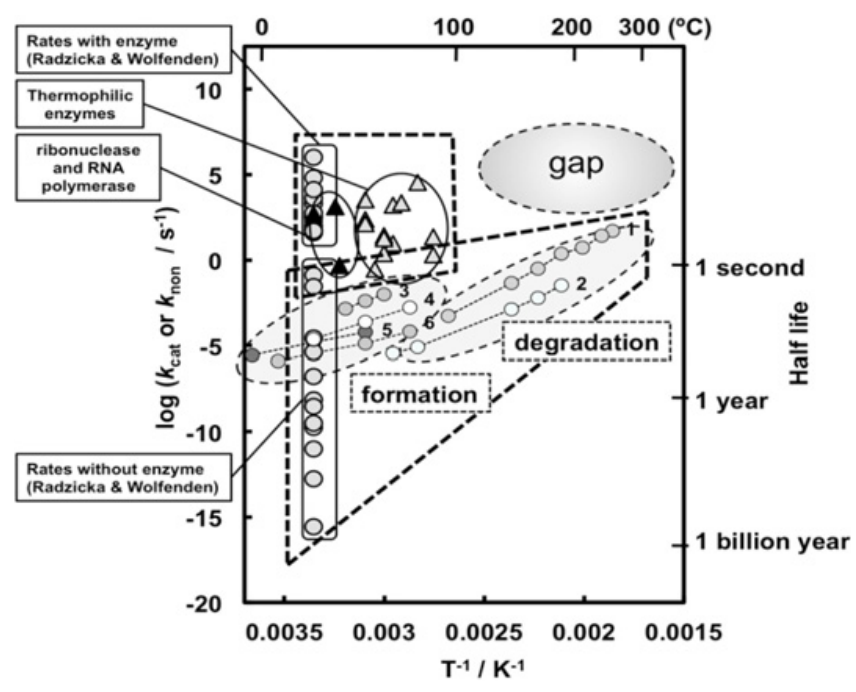

Figure 3. Comparison of the reaction rate with and without enzymes regarding prebiotic reactions. The horizontal axis indicates the inverse values of temperature $\left(\mathrm{T}^{-1}\right)$ and the vertical axis indicates logarithmic values of reaction rates. The numbers show the reaction rates determined by our previous studies. 1: adenosine 5'-triphosphate hydrolysis, 2: cleavage of dinucleotide, 3: 4-mer formation by template-directed reaction, 4: cyclization of hexanucleotide, 5: 4-mer formation by clay catalyzed reaction, 6: 3-mer formation by metal ion-catalyzed reaction. The gap indicates the temperature allowance for the emergence of primitive enzymes.

molecules form a crystalline matrix that can concentrate and organize mononucleotides in such a way that condensation reactions lead to ester bond synthesis. The amount of polymers synthesized was more than tenfold greater than that observed with lipid-enhanced reactions. The products synthesized are hydrolyzed when heated in alkaline $\mathrm{pH}$ ranges, and are partially hydrolyzed by RNase A. In summary, we showed that polymers that have physical and chemical properties of RNA can be synthesized by hydrothermal pools undergoing cycles of hydration and dehydration.

From another viewpoint, we frequently pointed out that the accumulation of biopolymers in a life-like system is determined by both the reaction rate of formation and degradation, and by the flow rate of inflow to, and outflow from the system. Thus, the accumulation of oligonucleotides is possible even at high temperatures and pressures if the relative formation or inflow rate is much higher than the degradation or outflow rate. Our kinetic measurements of prebiotic models (Fig. 1) predicted that the accumulation of oligonucleotides under hydrothermal conditions would be possible at high temperatures [28-31]. This is consistent with the fact that the formation and degradation of RNA molecules are maintained at temperatures up to $110-120^{\circ} \mathrm{C}$ in modern hyperthermophilic organisms.

In addition, the possibility of RNA formation is also supported based on the fact that the biochemical reactions are catalyzed by enzymes, such that the reaction rates are accelerated in the presence of enzymes as compared to the background reaction rates without enzymes [14, 31]. The principle was extrapolated to thermophilic organisms. Although we do not have much information about interactions and structures of ancient ribozymes and enzymes, we propose a possible role of temperature by comparing the enzymatic reaction rates and corresponding non-enzymatic reaction rates (Fig. 3). The gap between the enzymatic reaction rates and the corresponding non-enzymatic rates would reflect the possible enzymatic acceleration on the basis of a physico-chemical limitation. The large gap involving ancient catalytic activities consisting of RNA oligomers could have emerged at high temperatures from enzyme-like molecules possessing very weak activity and interaction with substrates.

In conclusion, we briefly correlate the RNA world hypothesis with hydrothermal environments. 


\section{ORIGINS}

\section{References}

[1] C. Woese, The Genetic Code, the Molecular Basis for Genetic Expression (Harper and Row, New York, 1967)

[2] F.H.C. Crick, J. Mol. Bio. 38, 67-379 (1968)

[3] L.E. Orgel, J. Mol. Bio. 38, 381-393 (1968)

[4] W. Gilbert, Nature 319, 618-618 (1986)

[5] R. Lohrmann, L.E. Orgel, Nature 244, 418-420 (1973)

[6] H. Sawai, J. Am. Chem. Soc. 98, 7037-7039 (1976)

[7] J.P. Ferris, G. Ertem, Science 257, 1387-1389 (1992)

[8] J.P. Ferris, A.R. Hill, J.R. Liu, L.E. Orgel, Nature 381, 59-61 (1996)

[9] A.D. Ellington, J.W. Szostak, Nature 346, 818-822 (1990)

[10] D.P. Bartel, J.W. Szostak, Science 261, 1411-1418 (1993)

[11] T. Inoue, L.E. Orgel, J. Mol. Bio. 162, 201-217 (1982)

[12] T. Inuoe, L.E. Orgel, Science 219, 859-862 (1983)

[13] A.R. Hill Jr., L.E. Orgel, T. Wu, Origins Life Evol. Biosph. 23, 285-290 (1993)

[14] K. Kawamura, Int. J. Astrobiol. 3, 301-309 (2004)

[15] K.A. Maher, D.J. Stevenson, Nature 331, 612-614 (1988)

[16] C. Sagan, G. Mullen, Science 177, 52-56 (1972)

[17] K. Kawamura, T. Nishi, T. Sakiyama, J. Am. Chem. Soc. 127, 522-523 (2005)

[18] K. Kawamura, Biochimie 94, 1441-1450 (2012)

[19] K. Kawamura, Bull. Chem. Soc. Jpn. 73, 1805-1811 (2000)

[20] K. Kawamura, Biochim. Biophys. Acta 1620, 199-210 (2003)

[21] N. El-Murr, M.-C. Maurel, M. Rihova, J. Vergne, G. Hervé, M. Kato, K. Kawamura, Naturwissenschaften 99, 731-738 (2012)

[22] K. Kawamura, H. Takeya, T. Kushibe, Y. Koizumi, Astrobiology 11, 461-469 (2011)

[23] S. Tobé, T. Heams, J. Vergne, G. Hervé, M.-C. Maurel, Nucl. Acid. Res. 33, 2557-2564 (2005)

[24] H. Kaddour, J. Vergne, G. Hervé, M.-C. Maurel, FEBS J. 278, 3739-3747 (2011)

[25] D. Deamer, S. Singaram, S. Rajamani, V. Kompanichenko, S. Guggenheim, Phil. Trans. Roy. Soc. Lond. B 361, 1809-1818 (2006)

[26] S. Rajamani, A. Vlassov, S. Benner, A. Coombs, F. Olasagasti, D. Deamer, Origins Life Evol. Biosph. 38, 57-74 (2008)

[27] L. Da Silva, M.-C. Maurel, D. Deamer, J Mol Evol 80, 86-97 (2015)

[28] K. Kawamura, M. Umehara, Bull. Chem. Soc. Jpn. 74, 927-935 (2001)

[29] K. Kawamura, N. Nakahara, F. Okamoto, N. Okuda, Viva Origino 31, 221-232 (2003)

[30] K. Kawamura, J. Maeda, J. Phys. Chem. A 112, 8015-8023 (2008)

[31] K. Kawamura, Proceedings of the Twelfth International Conference on the Simulation and Synthesis of Living Systems (H. Fellermann, M. Dörr, M. M. Hanczyc, L. L. Lauren, S. Maurer, D. Merkle, P.-A. Monnard, K. Støy, S. Rasmussen, Eds.), 37-44 (2010) 